\title{
NÚMERO IDEAL DE FILHOS E ARREPENDIMENTO PÓS-LAQUEADURA
}

Luiz Eduardo Campos de Carvalho, José Guilherme Cecatti*, Maria José Duarte Osis, Maria Helena de Sousa

Trabalho realizado no departamento de Tocoginecologia da Faculdade de Ciências Médicas da UNICAMP e Centro de Pesquisas em Saúde Reprodutiva de Campinas (CEMICAMP)

*Correspondência:

R. Alexander Fleming, 101 13081-970 Campinas, SP cecatti@unicamp.br

Fone/Fax: (19) 3788-9304

\begin{abstract}
RESUMO
OBjetivos. Avaliar a correlação entre o número ideal de filhos (NIF) e o arrependimento pós-laqueadura.

Métodos. Foi realizado um estudo de caso-controle aninhado a uma coorte. De um total de 3878 mulheres entrevistadas na coorte, 1012 estavam laqueadas no momento da entrevista do estudo original e constituíram a amostra deste estudo. Consideraram-se como arrependidas e se constituíram nos casos as mulheres que referiram que, nas mesmas circunstâncias, não voltariam a fazer a laqueadura (103 - 10,8\%). O NIF foi categorizado de acordo com sua relação com o número de filhos vivos (NV): NIF > NV e NIF $\leq$ NV. Calculou-se a proporção de mulheres arrependidas e estimou-se o risco de arrependimento conforme a relação NIF/NV por meio de odds ratio com os respectivos intervalos de confiança (IC) de $95 \%$. Posteriormente, estratificou-se a análise pelas variáveis de controle. Realizaram-se dois modelos de regressão logística múltipla para identificar os possíveis fatores de risco para o arrependimento pós-laqueadura entre as mulheres com NIF > NV.
\end{abstract}

RESULTADOS. Evidenciaram-se como fatores de risco independentes para o arrependimento pós-laqueadura o NIF $>$ NV $(\mathrm{OR} / 2,7)$, fazer a laqueadura com a intenção de esperar um tempo para ter mais filhos $(\mathrm{OR} 8,0)$ e ter tido mais de dois partos (OR 2,4).

ConcLusäo. Os dados do presente artigo sugerem que a avaliação prévia do NIF poderia auxiliar na identificação de um grupo de mulheres com maior risco para o arrependimento pós-laqueadura.

UnIteRMOs: Número ideal de filhos. Arrependimento pós-laqueadura. Planejamento familiar.

\section{INTRODUÇÃO}

Durante as últimas décadas, houve no Brasil um rápido crescimento na prevalência da laqueadura, que hoje é o método anticoncepcional mais utilizado, como pôde ser observado pela última Pesquisa Nacional sobre Demografia e Saúde (PNDS) de 1996, em que aproximadamente $40 \%$ das mulheres unidas, entre 15 e 49 anos, estavam laqueadas', proporção que era de $27 \%$ em 1986. No município de São Paulo, o índice de laqueadura passou de aproximadamente $7 \%$ em $1965^{2}$ para $26 \%$ no ano de $1988^{3}$. Para - Estado de São Paulo, a proporção de mulheres unidas entre I5 e 49 anos que estavam laqueadas era de 31,4\% em 1986 e passou a $33,6 \%$ anos em $1996^{4}$.

O crescimento da prevalência da esterilização cirúrgica feminina se deu em um contexto de pouca oferta e acesso aos métodos anticoncepcionais no Brasil, embora o Programa de Assistência Integral à Saúde da Mulher (PAISM) tenha sido adotado oficialmente pelo governo em 1983, estabelecendo, entre outras coisas, que a disponibilidade e o acesso aos meios de regulação da fecundidade eram essenciais para o atendimento integral à saúde das mulheres ${ }^{5,6}$. Essa deficiência tem produzido uma demanda não atendida de anticoncepção, que pode ser verificada, por exemplo, quando se estudam os resultados da última Pesquisa Nacional sobre Demografia e Saúde' e verifica-se que, entre as mulheres com mais de três filhos, o número ideal de filhos (NIF), em média, foi sempre inferior ao número observado de filhos. Nessa situação, é freqüente as mulheres se submeterem à esterilização cirúrgica com um número de filhos superior ao que desejariam ter tido ${ }^{7}$.

Durante muitos anos a esterilização feminina foi feita, de certa forma, na "clandestinidade", uma vez que a regulamentação para sua prática só ocorreu no final da década de $90 \mathrm{com}$ a Lei Ordinária n ${ }^{\circ}$ 9263, de junho de $1996^{8}, 9$. Essa situação contribuiu para que a laqueadura fosse realizada de maneira indiscriminada em mulheres jovens e com poucos filhos, associando-se ao crescimento nas taxas de cesariana e à cobrança, por alguns médicos, de um pagamento adicional, "por fora", para que o procedimento fosse realizado ${ }^{1,3,10}$.

O processo sócio-histórico em que ocorreu este aumento da prevalência da laqueadura também propiciou condições inadequadas para que as mulheres pudessem optar de maneira livre e informada por essa forma de regulação da fecundidade, o que, certamente, tem contribuído para o aparecimento de um contingente de mulheres arrependidas de terem realizado a laqueadura, em proporções que variam entre $10 \%$ e $20 \%{ }^{11-14}$. Muitas dessas mulheres acabam procurando os ambulatórios de esterilidade para solicitar a reversão cirúrgica da esterilização porque querem ter mais filhos ${ }^{15}$.

Dentre os fatores apontados como sendo de risco para o arrependimento pós-laqueadura, destacam-se a menor idade por ocasião da cirurgia, freqüentemente considerado como o mais relevante, menor número de filhos vivos, um relacionamento conjugal 
instável e/ou a mudança de companheiro, a realização da laqueadura durante uma cesariana ou no puerpério imediato, idade do último filho no momento da laqueadura, morte de um filho ou abortamentos e distúrbios menstruais $7,11,14-20$.

Nesse conjunto de possíveis fatores acima mencionados, não se encontram informações sobre qual seria a relação entre o número ideal de filhos (NIF), segundo a percepção das mulheres, e o risco de arrependimento pós-laqueadura. O conhecimento mais detalhado acerca dessa possível associação poderia contribuir para uma melhor avaliação e orientação das mulheres que solicitam a esterilização cirúrgica, visando minimizar as chances de arrependimento. O presente artigo tem como objetivo estudar a associação entre NIF e o arrependimento pós-laqueadura entre mulheres da cidade de Campinas, Estado de São Paulo.

\section{Métodos}

Foi realizado um estudo tipo caso-controle, não pareado, aninhado a uma coorte para avaliar a relação entre o número ideal de filhos e 0 arrependimento pós-laqueadura, utilizando-se secundariamente os dados obtidos de um estudo de coorte retrospectivo sobre a saúde reprodutiva das mulheres da cidade de Campinas, que tiveram o primeiro filho no ano de 1985, divididas segundo a via do primeiro parto: cesariana (expostas) e vaginal (não expostas), e avaliadas após um período de dez anos ${ }^{12,21,22}$.

$\mathrm{Na}$ análise deste artigo, utilizou-se apenas o grupo de mulheres laqueadas que haviam informado o NIF $(n=|0| 2)$, em que o arrependimento foi abordado como variável dependente (casos). Do questionário, a pergunta que foi considerada como a melhor para avaliar a questão do arrependimento foi "Se a Sra. tivesse hoje a mesma idade que tinha quando fez a laqueadura, voltaria a fazê-la?", pois era a que permitia detectar com maior fidedignidade a idéia do arrependimento, por situar a indagação no contexto de vida das entrevistadas por ocasião da laqueadura. As respostas a essa pergunta foram categorizadas em "não voltaria" e "voltaria/não sabe", que passaram a ser denominadas arrependimento: sim ou não, respectivamente. As mulheres que não sabiam esta resposta foram apenas 6 no total de 903 do grupo controle (inferior a 1\%) e, embora esse número fosse insuficiente para avaliar suas características, foram consideradas como não arrependidas para não superestimar o efeito de arrependimento.

Para melhor avaliar a relação entre o número ideal de filhos (NIF) e 0 arrependimento, definiu-se a primeira variável a partir da comparação entre o NIF e o número de filhos nascidos vivos (NV), obtendo-se duas categorias: NIF > NV e NIF $\leq$ NV. Para fins de análise estatística, neste artigo, a segunda categoria foi sempre considerada como referência.

Estimou-se, primeiramente, a porcentagem de mulheres arrependidas ao final de dez anos de seguimento da coorte. Foram calculados os odds ratio com os respectivos intervalos de confiança (IC) de $95 \%$, inicialmente apenas para relação NIF/NV e arrependimento. Posteriormente, a análise foi estratificada segundo as seguintes variáveis de controle: idade no momento da entrevista, escolaridade, estado marital, cor, trabalho, renda familiar, número de gestações, número de partos, número de abortos e nascidos mortos, idade quando foi laqueada, número de filhos quando laqueada, intenção de ter mais filhos, pagamento pela laqueadura, tipo de parto e motivos para fazer a laqueadura. Após a estratificação, desenvolveram-se dois modelos de regressão logística tendo como variável dependente o arrependimento pós-laqueadura. No primeiro, foram utilizadas as mesmas variáveis de controle, excluindo-se os motivos para fazer a laqueadura, e no segundo modelo utilizaram-se todas as variáveis.

O projeto de pesquisa que idealizou este estudo foi devidamente analisado e aprovado pela Comissão de Pesquisa do Departamento de Tocoginecologia/FCM e pelo Comitê de Ética em Pesquisa da FCM/ UNICAMP. Para análise dos dados foram utilizados dois pacotes estatísticos, SPSS e Epi.Info.

\section{Resultados}

Das 3878 mulheres originalmente entrevistadas, 1012 (26, I \%) estavam laqueadas e haviam informado o NIF, constituindo a amostra deste estudo. Destas, 109 referiram que não voltariam a fazer a laqueadura, 0 que representa uma proporção de arrependimento de 10,8\% e que constituíram os casos. As restantes 903 constituíram o grupo controle.

A maioria das mulheres que se declararam arrependidas apresentava idade inferior a 35 anos no momento da entrevista $(59,6 \%)$, escolaridade inferior a 8 a série $(59,6 \%)$, tinha companheiro (87,2\%), era da raça branca (62,4\%), trabalhava $(54,1 \%$ ) e referia renda familiar acima de oito salários mínimos (57,7). Notou-se ainda que, neste grupo, no momento da laqueadura, 68,8\% das mulheres tinham até 30 anos, mais de metade possuía até dois filhos, $84,4 \%$ não pretendiam ter mais filhos, cerca de $25 \%$ pagaram pela esterilização e quase $80 \%$ foram laqueadas durante uma cesariana. Elas diferiram significativamente do grupo de não arrependidas pela maior proporção sem companheiro, com idade menor que 30 anos e menor proporção que não tinha intenção de ter mais filhos.

A Tabela I mostra que o grupo de mulheres laqueadas com o NIF $>$ NV, de uma maneira geral, apresentou um risco quase 12 vezes maior de se arrepender de ter feito a laqueadura do que aquelas com o NIF $\leq N V$. Este risco foi ainda mais evidente especialmente nos seguintes grupos: com idade inferior a 35 anos $(\mathrm{OR} \quad 17,0)$, que estudaram até $8^{a}$ série do ensino fundamental (OR 17,3), que tinham companheiro $(O R \mid 3,1)$, que não eram brancas (OR 26,7), que não trabalhavam (OR 20,2), que tinham renda familiar mensal de até oito salários mínimos $(O R \mid 3,0)$, que tinham até duas gestações ou partos (OR em torno de 15,0) e que não declararam abortamentos (OR I2, I).

Os resultados apresentados na Tabela 2 apontam, ainda, que a chance de arrependimento para mulheres com NIF>NV foi superior entre as com idade inferior a 30 anos (OR 16,8) por ocasião da cirurgia, que tinham mais de dois filhos nessa época $(\mathrm{OR}$ 16,2), que declararam que, quando foram operadas, sua intenção era esperar um pouco para ter mais filhos (OR 4I,2), que pagaram pela laqueadura $(O R$ I2,4) e que foram esterilizadas durante parto normal ou em outra circunstância que não uma cesárea $(O R$ 15,4). Ao se avaliarem os motivos pelos quais as mulheres se submeteram à esterilização cirúrgica, percebeu-se que $o$ risco de arrependimento foi maior para aquelas que 


\begin{tabular}{|c|c|c|c|c|c|c|}
\hline \multicolumn{7}{|c|}{$\begin{array}{c}\text { Tabela I - Porcentagem de mulheres com NIF > NV segundo } \\
\text { arrependimento, e estratificação por características } \\
\text { sociodemográficas ereprodutivas }\end{array}$} \\
\hline \multirow[b]{3}{*}{ Característica } & \multicolumn{4}{|c|}{ Arrependimento } & \multirow[b]{3}{*}{ OR } & \multirow[b]{3}{*}{ IC $95 \%$} \\
\hline & \multicolumn{2}{|c|}{ Sim } & \multicolumn{2}{|c|}{ Não } & & \\
\hline & $(\%)$ & $(n)$ & $(\%)$ & $(n)$ & & \\
\hline \multicolumn{7}{|l|}{ Relação NIF/NV } \\
\hline$N I F>N V$ & 52,3 & $(109)$ & 8,4 & (903) & 11,9 & $7,5-19,0$ \\
\hline \multicolumn{7}{|l|}{ Idade } \\
\hline$<35$ anos & 55,4 & $(65)$ & 6,8 & $(5 \mid 4)$ & 17,0 & $9,0-32,3$ \\
\hline$\geq 35$ anos & 47,7 & $(44)$ & 10,5 & (389) & 7,7 & $3,8-16,0$ \\
\hline E & & & & & $11,9 \#$ & $7,6-19,4$ \\
\hline \multicolumn{7}{|l|}{ Escolaridade } \\
\hline Até $8^{\mathrm{a}}$ série & 63,1 & (65) & 9,0 & $(50 I)$ & 17,3 & $9,2-32,6$ \\
\hline$>8^{\mathrm{a}}$ série & 36,4 & (44) & 7,7 & (402) & 6,8 & $3,2-14,8$ \\
\hline & & & & & 12,0\# & $7,5-19,1$ \\
\hline \multicolumn{7}{|l|}{ Estado marital } \\
\hline Sem companheiro & 42,9 & $(14)$ & 13,0 & $(54)$ & 5,0 & $1,1-23,3$ \\
\hline Com companheiro & 53,7 & (95) & 8,1 & (849) & $|3|$, & $8,0-21,6$ \\
\hline & & & & & $11,5 \#$ & $7,4-18,9$ \\
\hline \multicolumn{7}{|l|}{ Cor da pele ${ }^{\S}$} \\
\hline Branca & 42,6 & (68) & 8,9 & (582) & 7,6 & $4,2-13,8$ \\
\hline Outra & 68,3 & $(4 I)$ & 7,5 & $(321)$ & 26,7 & $11,5-62,9$ \\
\hline & & & & & $11,7 \#$ & $7,5-19,0$ \\
\hline \multicolumn{7}{|l|}{ Trabalho } \\
\hline Não trabalha & 62,0 & (50) & 7,5 & $(454)$ & 20,2 & $9,8-41,7$ \\
\hline \multirow[t]{2}{*}{ Trabalha } & 44,1 & (59) & 9,4 & (449) & 7,6 & $4,0-14,6$ \\
\hline & & & & & $11,6 \#$ & $7,5-\mid 9,1$ \\
\hline \multicolumn{7}{|l|}{ Renda familiar* } \\
\hline Até 8SM & 54,5 & $(44)$ & 8,5 & (307) & 13,0 & $6,0-28,3$ \\
\hline \multirow[t]{2}{*}{$>8 S M$} & 50,0 & (60) & 8,2 & (527) & 11,3 & $6,0-21,3$ \\
\hline & & & & & $11,9 \#$ & $7,4-19,3$ \\
\hline \multicolumn{7}{|c|}{ Número de gestações } \\
\hline Até 2 & 67,4 & $(46)$ & 11,7 & $(428)$ & $\mid 5,6$ & $7,5-32,8$ \\
\hline$>2$ & 41,3 & (63) & 5,5 & (475) & $|2|$, & $6,1-24,2$ \\
\hline & & & & & $13,8 \#$ & $8,4-22,4$ \\
\hline \multicolumn{7}{|c|}{ Número de partos } \\
\hline Até 2 & 66,7 & (63) & $\mid 1,4$ & (569) & $\mid 5,5$ & $8,3-29,0$ \\
\hline$>2$ & 32,6 & (46) & 3,3 & (334) & 14,2 & $5,6-36,7$ \\
\hline & & & & & $15, \mid \#$ & $9,1-25,2$ \\
\hline \multicolumn{7}{|c|}{ Número de abortos } \\
\hline Nenhum & 51,2 & $(84)$ & 8,0 & (687) & $|2|$, & $7,0-20,7$ \\
\hline \multirow[t]{2}{*}{$\geq \mid$} & 56,0 & $(25)$ & 9,7 & $(216)$ & 11,8 & $4,4-32,4$ \\
\hline & & & & & $12,0 \#$ & $7,5-19,2$ \\
\hline \multicolumn{7}{|c|}{$\mathbf{N}^{0}$ nascidos mortos } \\
\hline Nenhum & 52,3 & $(109)$ & 8,3 & $(891)$ & $|2|$, & $7,6-19,4$ \\
\hline$\geq 1$ & 0,0 & $(0)$ & 16,7 & $(12)$ & $\varnothing$ & \\
\hline \multicolumn{3}{|c|}{ Total de mulheres $(\mathbf{n})(109)$} & (903) & & & \\
\hline $\begin{array}{l}\text { * Faltou informação de } \\
\text { \# OR de Mantel-Hae } \\
\text { §teste de homogeneic } \\
\text { ø Não foi possível o cá }\end{array}$ & mulheres & 0,05 & & & & \\
\hline
\end{tabular}

Tabela 2 - Porcentagem de mulheres com NIF > NV segundo arrependimento, estratificada por circunstâncias no momento da laqueadura e por motivos para fazer a cirurgia

\begin{tabular}{|c|c|c|c|c|c|c|}
\hline \multirow[b]{3}{*}{ Circunstâncias } & \multicolumn{4}{|c|}{ Arrependimento } & \multirow[b]{3}{*}{ OR } & \multirow[b]{3}{*}{ IC $95 \%$} \\
\hline & \multicolumn{2}{|c|}{ Sim } & \multicolumn{2}{|c|}{ Não } & & \\
\hline & $(\%)$ & (n) & $(\%)$ & $(n)$ & & \\
\hline \multicolumn{7}{|c|}{ Idade quando laqueada } \\
\hline$<30$ anos & 57,3 & (75) & 7,4 & $(486)$ & 16,8 & $9,2-31,0$ \\
\hline \multirow[t]{2}{*}{$\geq 30$ anos } & 41,2 & (34) & 9,6 & $(4 \mid 6)$ & 6,6 & $2,9-14,9$ \\
\hline & & & & & $12,1 \#$ & $7,5-19,3$ \\
\hline \multicolumn{7}{|c|}{$\mathrm{N}^{0}$ de filhos quando laqueada } \\
\hline Até 2 & 64,1 & (64) & 11,4 & $(568)$ & 13,8 & $7,5-25,5$ \\
\hline \multirow[t]{2}{*}{$>2$} & 35,6 & $(45)$ & 3,3 & (335) & 16,2 & $6,4-41,8$ \\
\hline & & & & & |4,4\# & $8,8-24,1$ \\
\hline \multicolumn{7}{|c|}{ Intenção por mais filhos } \\
\hline Não tinha intenção & 45,7 & (92) & 8,3 & (890) & 9,3 & $5,6-15,3$ \\
\hline \multirow[t]{2}{*}{ Queria esperar } & 88,2 & $(17)$ & 15,4 & $(13)$ & 41,2 & $3,8-762,8$ \\
\hline & & & & & $10,4 \#$ & $6,3-16,4$ \\
\hline \multicolumn{7}{|c|}{ Pagamento de cirurgia } \\
\hline Sim & 55,6 & (27) & 9,1 & $(186)$ & 12,4 & $4,6-34,2$ \\
\hline \multirow[t]{2}{*}{ Não } & 51,2 & (82) & 8,2 & $(7 \mid 7)$ & 11,7 & $6,8-20,1$ \\
\hline & & & & & 11,9\# & $7,4-19,0$ \\
\hline
\end{tabular}

Tipo de parto

$\begin{array}{lllllll}\text { Cesárea } & 50,6 & (87) & 8,4 & (728) & 11,2 & 6,6-18,9 \\ \text { Parto normal/outra } & 59,1 & (22) & 8,6 & (175) & 15,4 & 5,1-47,6 \\ \text { ocasião } & & & & & & \end{array}$

\section{Motivos para fazer a cirurgia \\ Muitos filhos / NIF}

\begin{tabular}{lllllll} 
Sim & 35,3 & $(5 \mid)$ & 5,7 & $(662)$ & 9,0 & $4,4-18,2$ \\
Não & 71,1 & $(38)$ & 14,6 & $(199)$ & $\mid 4,4$ & $6,0-35,0$ \\
& & & & & $\mid 1,3 \#$ & $6,4-18,5$ \\
\hline
\end{tabular}

\begin{tabular}{lllllll}
\hline Dificuldades financeiras & & & & & & \\
Sim & 60,0 & $(20)$ & 6,6 & $(196)$ & 21,1 & $6,6-70,1$ \\
Não & 47,8 & $(69)$ & 8,1 & $(665)$ & 10,4 & $5,8-18,6$ \\
& & & & & $12,0 \#$ & $7,3-20,3$ \\
\hline
\end{tabular}

\section{Problemas na gravidez}

$\begin{array}{lllllll}\text { Sim } & 58,3 & (12) & 11,6 & (121) & 10,7 & 2,6-46,3 \\ \text { Não } & 49,4 & (77) & 7,2 & (740) & 12,6 & 7,2-22,1 \\ & & & & & 12,3 \# & 7,4-20,7\end{array}$

\section{Outros motivos}

\begin{tabular}{lllllll} 
Sim & 56,3 & $(32)$ & 10,3 & $(302)$ & 11,2 & $4,8-26,8$ \\
Não & 47,4 & $(57)$ & 6,4 & $(559)$ & 13,1 & $6,7-25,5$ \\
& & & & & $\mid 2,3 \#$ & $7,4-20,7$ \\
\hline
\end{tabular}

Total de mulheres $(\mathrm{n}) \quad$ (109) (903)

\# OR de Mantel-Haenszel 


\section{Tabela 3 - Variáveis associadas ao arrependimento. Análise} de regressão logística múltipla

\begin{tabular}{|c|c|c|c|c|c|}
\hline Variável & Coef. & EP coef. & $\mathrm{p}$ & OR & IC $95 \%$ p/ OR \\
\hline \multicolumn{6}{|l|}{ Modelo I $(n=938)$} \\
\hline NIFversus NV (NIF>NV) & 2,54 & 0,26 & $<0,001$ & 12,7 & $7,6-21,2$ \\
\hline $\begin{array}{l}\text { Intenção de ter mais filhos } \\
\text { (queria esperar) }\end{array}$ & 2,08 & 0,48 & $<0,001$ & 8,0 & $3,1-20,6$ \\
\hline Número de partos (> 2) & 0,86 & 0,26 & $<0,001$ & 2,4 & $1,4-3,9$ \\
\hline Estado marital (com comp.) & $-0,84$ & 0,39 & 0,031 & 0,4 & $0,2-0,9$ \\
\hline Constante & $-2,45$ & 0,40 & $<0,001$ & & \\
\hline \multicolumn{6}{|l|}{ Modelo 2 $(n=883)$} \\
\hline NIFversus NV (NIF $>N V$ ) & 2,62 & 0,28 & $<0,001$ & 13,7 & $8,0-23,6$ \\
\hline $\begin{array}{l}\text { Intenção em ter mais filhos } \\
\text { (queria esperar) }\end{array}$ & 2,07 & 0,69 & 0,003 & 7,9 & $2,0-30,5$ \\
\hline Número de partos (> 2) & 0,68 & 0,27 & 0,012 & 2,0 & $1,2-3,4$ \\
\hline Constante & $-3,22$ & 0,22 & $<0,001$ & & \\
\hline
\end{tabular}

mencionaram que tinham dificuldades financeiras (OR 21,1$)$. 0 risco foi menor quando o motivo apontado foi já ter muitos filhos (OR 9,0) ou problemas na gravidez (OR 10,7) (Tabela 2).

Os modelos de regressão logística múltipla apontaram como fatores de risco independentes para o arrependimento pós-laqueadura o NIF > NV (OR em torno de 12,0), mais de dois partos (OR próximo de 2,0 ) e a intenção, no momento da cirurgia, de esperar um pouco para ter mais filhos (OR de 8,0) (Tabela 3).

\section{Discussão}

Os resultados apresentados indicaram que o grupo de mulheres que tinham NIF > NV apresentou um risco maior de arrependimento se comparado ao das mulheres com NIF $\leq N V$, o que soa como óbvio, já que isto significa que essas mulheres foram esterilizadas sem ter atingido o número de filhos que gostariam de ter tido. Cabe, então, responder primeiramente por que essas mulheres optaram por um método permanente, uma vez que seria lógico imaginar que deveriam ter optado por um MAC reversível que, de fato, lhes permitisse esperar um pouco antes de ter mais filhos.

É preciso lembrar que a opção dessas mulheres pela laqueadura se deu em uma época em que a esterilização era realizada, freqüentemente, de forma indiscriminada, sem qualquer regulamentação oficial|23,24. Isto resultava em que as mulheres, de modo geral, não recebessem orientação adequada acerca da laqueadura e das possíveis alternativas contraceptivas ${ }^{14}$. Nessa situação, a opção pela laqueadura não se dava de maneira livre e informada, o que ficou claro neste artigo, uma vez que muitas dessas mulheres, que não haviam alcançado o seu NIF, submeteram-se à laqueadura com a intenção de espaçar os nascimentos, pois declararam que queriam esperar um pouco antes de ter mais filhos.

É possível aprofundar essa discussão acerca da opção pela laqueadura não ter sido livre e informada, aumentando o risco de arrependimento, quando se observa que no grupo de mulheres com $\mathrm{NIF}>\mathrm{NV}$ foram fatores de risco, ou marcadores para o arrependimento, a escolaridade até o nível fundamental, a cor da pele não ser branca, não realizar trabalho remunerado, a menor renda familiar e a realização da laqueadura em vista de dificuldades financeiras. Essas são características de mulheres que, certamente, pertenciam a um menor nível socioeconômico, cujo acesso aos diferentes meios de regulação de fecundidade é mais restrito. Nesse contexto, em uma determinada época da vida dessas mulheres, a laqueadura acaba se configurando como a única alternativa contraceptiva ${ }^{7}$. Elas têm que resolver o problema urgente de não poder ter mais filhos naquele momento, o que não significa que, de fato, não querem nunca mais ter filhos. Isto já tem sido discutido em outros estudos que focalizaram o risco de arrependimento pós-laqueadura, e que salientaram que a menor idade por ocasião da esterilização é um fator de risco por excelência porque implica nas possibilidades de mudanças na vida das mulheres que, por vezes, acabam viabilizando novas gestações, tais como mudanças de parceiro e de situação econômica ${ }^{7,13,14,16}$.

Um outro resultado deste estudo, que corrobora a discussão acima, é o maior risco de arrependimento das mulheres com NIF $>$ NV e que tinham mais de dois partos. Aparentemente, isto poderia ser uma contradição, porém, também pode indicar que essas são mulheres que estariam como que predispostas a ter mais filhos, desejariam tê-los, mas acabam optando pela esterilização por não saber como regular a fecundidade eficazmente de outra maneira ${ }^{25}$.

Por outro lado, as mulheres que disseram ter optado pela laqueadura devido a um número elevado de filhos, ou por problemas durante a gravidez, ou que não apresentavam dificuldades financeiras, tiveram um menor risco de arrependimento, o que também já foi evidenciado em outros trabalhos ${ }^{11,14}$.

Embora não identificados na análise múltipla, alguns resultados deste estudo aparentemente não confirmam dados já estudados por outros autores. Os nossos resultados evidenciaram que entre as mulheres com NIF > NV onde a laqueadura foi realizada em um procedimento que não uma cesariana e mediante pagamento, a chance de arrependimento foi maior. A primeira variável aparentemente contradiz a literatura, que correlaciona uma menor chance de arrependimento com a realização da laqueadura fora do ciclo gravídicopueperal| ${ }^{16,17,19}$. Este é um achado que mereceria um estudo posterior mais aprofundado para tentar encontrar uma explicação plausível. Cabe salientar, ainda, que a maioria das laqueaduras (próximo de 80\%) foi realizada durante uma cesariana tanto nos casos como nos controles e, na época do estudo, este procedimento freqüentemente estava associado ao pagamento "por fora".

\section{Conclusão}

Cabe refletir, ainda, se a realidade indicada pelos resultados aqui discutidos tem mudado ou não, uma vez que, desde 1997, a realização da laqueadura está normatizada pelo Ministério da Saúde, enfatizandose a necessidade de garantir que a opção por essa forma de contracepção seja feita de maneira, de fato, livre e informada. De acordo com o espírito da Lei $n^{\circ} 9263$, os esforços atuais devem se concentrar em aumentar a oferta e variedade dos MAC, bem como 
viabilizar a orientação em planejamento familiar o mais precocemente possível, para que todas as mulheres, desde a adolescência, possam ter a autonomia para decidir sobre o seu futuro reprodutivo. Nesse sentido, os resultados do presente artigo, apesar de refletirem uma época anterior à legislação da laqueadura, apontam para a necessidade de avaliar com as mulheres a questão do NIF no momento de optar por um método contraceptivo.

\section{Conflito de interesse: não há}

\section{SUMMARY}

\section{IDEAL NUMBER OF CHILDREN AND REGRET AFTER TUBAL LIGATION IN A COHORT OF WOMEN}

OBJECTIVES. The purpose of this paper was to evaluate the relationship between the ideal number of children (INC) and post tubal ligation regret.

METHODS. A nested case-control study was carried out with a total of 3878 women interviewed. Ofthese 1012 had been surgically sterilized at the time of the interview of the original study and as such comprise the sample of this study. These are constituted by women who stated that they would not undergo tubal ligation again and who regretted the procedure $(103-10.8 \%)$ if they were to face the same circumstances. The Ideal Number of Children INC- was divided into two groups according to the relationship with the number of live births $(L B): I N C>\angle B$ and INC $\leq L B$. The proportion of women who regretted was calculated and the risk of regret estimated according to the relation INC/LB by means of Odds Ratios with the respective $95 \%$ confidence interval. Then the analysis was stratified according to control variables. Two multiple logistic regression models were developed in order to identify the independent risk factors associated with regretamong women with INC $>\angle B$.

RESULTS. The independent risk factors identified for post tubal ligation regret are INC $>\angle B(O R=12.7)$, for performance of tubal ligation with the intention of just waiting some time before having more children $(O R=8.0)$ and for having had more than two deliveries at the time of sterilization $(O R=2.4)$.

CONCLUSION. Results suggest thata previous evaluation of the INC could help identify women with a higher risk for post ligation regret. [Rev Assoc Med Bras 2006; 52(5): 293-7]

KEY wORDS: Ideal Number of Children. Surgical sterilization regret. Family planning.

\section{REFERÊNCIAS}

I. Bemfam. Sociedade Civil Bem Estar Familiar no Brasil, Macro Internacional Programa de Pesquisas de Demografia e Saúde (DHS). Pesquisa nacional sobre demografia, 1996. Rio de Janeiro: Macro Internacional Inc; 1997.

2. Berquó E, Oya DT. A esterilização feminina. In: Berquó E, Oliveira MCAF, Camargo CPF, editores. A fecundidade em São Paulo: características demográficas e sócio-econômicas. São Paulo: CEBRAP/Ediora Brasileira de Ciências; 1977. p.453-64.

3. Osis MJD, Hardy E, Simões IR, Vera S, Faúndes A. Laqueadura tubárea nos serviços de saúde do Estado de São Paulo. Rev. Ginecol. Obst 1990; I: 195-204.

4. Vieira EM, Badiani R, Dal Fabbro AL, Rodrigues Júnior A. Características do uso de métodos anticoncepcionais no Estado de São Paulo. Rev Saúde Pública 200 I ;36:263-70.
5. Giffin K. Women's health and the privatization of fertility control in Brazil. Soc Sci Med 1994:39:355-60.

6. Osis MJD. PAISM: um marco na abordagem da saúde reprodutiva no Brasil. Cad Saúde Pública 1998; I 4(Supl I):25-32.

7. Osis MJD, Faundes A, Sousa MH, Duarte GA, Bailey P. Fertility and reproductive history of sterilized and non-sterilized women in Campinas, São Paulo, Brazil. Cad Saúde Pública 2003; 19: I09-18.

8. Brasil, 1997. Lei ordinária no 9263 de 12 de janeiro de 1996. Regula o parágrafo 7 do artigo 226 da Constituição Federal, que trata do planejamento familiar, estabelece penalidades e dá outras providências. Partes vetadas correspondentes aos artigos 10, II, I4 e 15. Diário Oficial da União, Brasília, 20 ago 1997. p. 17989, col. I.

9. Brasil, 1997. Ministério da Saúde/Secretaria da Assistência à Saúde. Portaria n. 144, de 20 de novembro de 1997. Diário Oficial da União, Brasília, 24 nov 1997, n. 227, seção I, p.27409.

10. Osis MJD, Hardy E, Simões IR, Vera S, Faúndes A. Laqueadura tubária nos serviços de saúde do Estado de São Paulo. Rev Ginecol Obstet 1990; I:195-204.

I I. Barbosa CP. Avaliação do grau de insatisfação pós-laqueadura em São Bernardo do Campo. Reprodução 1994;9:159-62.

12. Besteti Pires HM. O impacto das altas taxas de cesárea sobre a fecundidade de uma população. Um estudo de coorte retrospectivo em Campinas, Brasil [tese]. Campinas: Faculdade de Ciências Médicas/ UNICAMP; 2000

13. Osis MJD, Faúndes A, Sousa MH, Bailey P. Conseqüências do uso de métodos anticoncepcionais na vida das mulheres: o caso da laqueadura tubária. Cad Saúde Pública 1999; | 5:521-32.

14. Vieira EM, $O$ arrependimento após a esterilização feminina. Cad Saúde Pública 1998: I 4(Supl I):59-68.

15. Bahamondes L, Petta CA, Faúndes A, Dias J, Bedone A. Significado do recente aumento do número de solicitações de reversão de laqueadura em um serviço de esterilidade. Femina 1992;20:360-2.

16. Hardy E, Bahamondes L, Osis MJD, Costa RG, Faúndes A. Risk factors for tubal sterilization regret, before surgery. Contraception 1996;54: 1 59-62.

17. Hillis SD, Polly AM, Herbert BP. Post sterilization regret: findings from the United States collaborative review of sterilization. Obstet Gynecol 1999:93:889-95.

18. Minella LS. Aspectos positivos e negativos da esterilização tubária do ponto de vista de mulheres esterilizadas. Cad Saúde Pública | 998; | 4(Sup| I):69-79.

19. Schimidt JE, Hillis SD, Polly AM, Gary J, Herbert BP. Requesting information about and obtaining reversal after tubal ligation sterilization: findings from the $U$. S. collaborative review of sterilization. Fertil Steril 2000:74:892-98.

20. Wilcox LS, Chu SY, Eaker ED, Zeger SL, Peterson HB. Risk factors for regret after tubal sterilization: 5 years of follow-up in a prospective study. Fertil Steril 1991;55:927-33.

21. Carvalho LEC, Cecatti J G, Osis MJD, Sousa MH. Número ideal de filhos como fator de risco para laqueadura tubária. Cad Saúde Publica 2004;20: I565-74.

22. Cecatti JG, Pires HM, Faundes A, Duarte Osis MJ. Factors associated with vaginal birth after previous cesarean section in Brazilian women. Rev Panam Salud Publica 2005; | 8: I 07- 13.

23. Faria VE. Políticas de governo e regulação de fecundidade: conseqüências não antecipadas e efeitos perversos. Ciências Sociais Hoje 1989;62- 103.

24. Martine G. Brazil's fertility decline, 1965-95: a fresh look at key factors. Pop Develop Rev 1996;22:47-75.

25. Serruya S. Mulheres esterilizadas. Submissão e desejo. Belém: NAEA UPFA/UEPA; 1996. 